\title{
Remote hearing aid fitting: Tele-audiology in the context of Brazilian Public Policy
}

Silvio Pires Penteado', Sueli de Lima Ramos², Linamara Rizzo Battistella 3 , Silvio Antonio Monteiro Marone ${ }^{4}$, Ricardo Ferreira Bento5.

1) $\mathrm{PhD}$ in Sciences. Full-time Researcher, Fundação Otorrinolaringologia.

2) $\mathrm{PhD}$ from the Federal University of São Paulo. Clinical Head, Instituto Sul Mineiro de Otorrinolaringologia.

3) Legal Medicine, Medical Ethics, Social Medicine Department, Universidade de São Paulo (USP), São Paulo, Brazil.

4) Deputy Professor, Medical School, University of São Paulo. Deputy Professor, Medical School, University of São Paulo.

5) Professor and Chairman, Otorhinolaryngology Department, Medical School, University of São Paulo. Professor and Chairman, Otorhinolaryngology Department, Medical School, University of São Paulo.

Institution: Faculdade de Medicina da Universidade de São Paulo. São Paulo / SP - Brazil.

Mailing address: Ricardo Ferreira Bento - Avenida Dr. Enéas de Carvalho Aguiar, 255 - 6 Floor - Room 6167 - São Paulo / SP- Brazil - E-mail: rbento@ gmail.com Article received in March 4, 2012. Article approved in May 8, 2012.

\section{SUMMARY}

Introduction: Currently, the Brazilian government has certificated nearly 140 specialized centers in hearing aid fittings through the Brazilian National Health System (SUS). Remote fitting through the Internet can allow a broader and more efficient coverage with a higher likelihood of success for patients covered by the SUS, as they can receive fittings from their own homes instead of going to the few and distant specialized centers.

Aim: To describe a case of remote fitting between 2 cities, with revision of the literature.

Method: Computer gears, a universal interface, and hearing aids were used. Case study: An audiologist located in a specialized center introduced a new hearing aid and its fitting procedure to a remote center ( $200 \mathrm{~km}$ away). The specialized center helped the remote center in fitting a hearing aid in 2 patients, and performed fitting in one of its own patients. The whole process was done through the Internet with audio and video in real time.

Results: Three patients were fitted remotely. Three audiologists were remotely trained on how to fit the hearing aids.

Conclusions: Remote fitting of hearing aids is possible through the Internet, as well as further supplying technical training to a remote center about the fitting procedures. Such a technological approach can help the government advance public policies on hearing rehabilitation, as patients can be motivated about maintaining their use of hearing aids with the option to ask for help in the comfort of their own homes.

Keywords: public policies, hearing aids, telemedicine, internet, technological development, biomedical engineering.

\section{INTRODUCTION}

Sullivan and Lugg, cited by Maheu et al. (1), recorded one of the pioneering Telemedicine events, which occurred through radio communication in Antarctica in the early twentieth century. In 1965, after the launch of the first intercontinental satellite, DeBAKEY performed a surgical aortic valve replacement at Methodist Hospital in Houston, Texas (USA) while being observed by his colleagues among the Geneva University Medical Faculty in Geneva, Switzerland (2). Puentes et al. (3) note that Internet availability has permitted rapid development of centers for specialized education of patients, discussion forums, online publications, and specialized journals, which allow the dissemination and exchange of information between specialists and non-specialists.

Chorbev and Mihajlov (4) have concluded that recent telemedicine applications incorporate satellites to connect centers of knowledge in various regions of the globe in a quick, reliable, and secure way that is affordable in many branches of medicine.

There are several definitions and purposes of telemedicine practice. The U.S. Department of Health and Human Services (5) describes telemedicine as "the use of telecommunications technology for medical diagnosis, monitoring and therapeutic purposes when distance separates the users." As Тонме et al. (6) assert, telemedicine "should provide patients and practitioners located at remote sites the possibility to access primary or specialty care thereby increasing the quality of care while minimizing costs." BeLARdinelil et al. (7) have demonstrated the effects of the increasing diffusion of computer technology and telecommunications, which have made the "telemedicine approach, the providing of medical information and services at a distance, a fundamental tool to harmonize quality and cost in the Health System." Wоotтon (8) describes telemedicine as "a technique that has been used in 
industrialized countries, such as North America and Australia, for bringing health care to rural and remote areas where there are few doctors or other health-care workers." From the engineering perspective, there are many applications for telemedicine throughout the specialties, including cardiology (9-11), surgery (12-14), dermatology (15-17), emergency medicine (18-20), speech therapy (21-24), gynecology (25-27), immunology (28-29), neurology (3032), ophthalmology (33-36), pathology (37-40), psychiatry (41-42), radiology (43-46), otolaryngology (47-49), and others.

The World Health Organization (WHO) describes hearing loss as an epidemic. In certain countries, hearing loss disabilities, i.e., disabilities involving thresholds above $40 \mathrm{~dB}$ in adults or $30 \mathrm{~dB}$ in children, can affect up to $16 \%$ of the population (50). The WHO estimates that the best hearing health policies are related to primary prevention activities (51-52). Every hearing impaired person is a candidate for an electronic hearing aid, which is an electronic device for selective amplification intended to minimize the effects of hearing loss when it is not possible by other medical or surgical means (53). An electronic hearing aid can attenuate even hearing losses classified as severe. Cochlear implants are indicated for cases of profound hearing loss (54-55).

The WHO notes that due to lack of infrastructure, services for hearing rehabilitation in developing countries are more difficult to manage when compared to developed countries (56). Goulios and PatuZZi (57) conducted a study in 2008 that included $78 \%$ of the worldwide population; this study showed that Brazil has 6,971 inhabitants per audiologist, lower than the number in the USA $(19,603)$, Australia $(15,178)$, Sweden $(12,055)$, and Israel $(11,208)$, though this number was lower than that reported for Argentina $(5,490)$. This same research reported significant numbers of inhabitants peraudiologist in Mexico $(258,643)$, Botswana $(297,500)$, Russia $(477,487)$, India $(1,065,462)$, Thailand $(1,243,860)$, Libya $(2,775,500)$, and Pakistan $(21,939,714)$. Goulios and Patuzzi stated that Brazil has 29,745 inhabitants per otolaryngologist, fewer than that in Costa Rica (55,640), Nicaragua (273,300), Indonesia $(362,843)$, and Cambodia $(282,880)$, but more than that in Italy $(12,761)$, Denmark $(15,326)$, Sweden $(16,681)$, or the USA $(24,504)$. In some African countries like Nigeria (1/ $2,066,817)$ and Madagascar $(1 / 3,480,800)$, the otolaryngologist/population marks are impressive.

KOKESH et al. (58) registered an important role for telemedicine in otology, providing more efficient access to appointments and post-surgical care, e.g., for conducting positioning and exchange of tympanostomy tubes in a native population living in remote Alaska. This population benefitted from the convenience of care services provided closer to their homes and reduced transportation costs, showing higher rates of acceptance among treatments.

KrUmm et al. (59) have enumerated the positive effects of diagnostic tests such as distortion product otoacoustic emissions (DPOAEs), brainstem-evoked response audiometry (BERA), spontaneous otoacoustic emissions (SOAEs), and audiometry (60) in countryside populations in the USA. Bloom (61) stated that a specialized team using satellite clinics in the region of Honolulu saved about \$25 million (US) compared with face-to-face consultation. At the end of 2003, Givens et al. (62) reported that results of remote hearing loss evaluations obtained in a double-blind test including 31 adult patients were validated against personal audiology evaluation, registering threshold increases within $1.3 \mathrm{~dB}$. In late 2003, Givens and Elangovan (63) repeated the tests in the same double-blind format with 45 patients and validated it against face-to-face audiology consultation, registering a variation of $1.3 \mathrm{~dB}$ by bone-conduction audiometry and 1.2 $\mathrm{dB}$ by pure tone audiometry.

RAmos et al. (64) narrated the success of Telemedicine to fit individuals with cochlear implants, concluding that "programming is feasible, safe, it has simple interface and a proven cost-effectiveness." In the XIX Congress of the International Federation of Oto-Rhino-Laryngological Societies (IFOS), research presented by the Institute of Physiology and Pathology of Hearing in Warsaw reported that 29 patients were attended through telemedicine by the research team, concluding that "the adjustments in cochlear implants have proven to be feasible, safe, approved by patients and healthcare specialists" and "it reduces costs and travelling time and provides the comfort of being treated in locations nearby their homes" (65). Zumpano et al. (66) demonstrated the functioning of telemedicine with cochlear implants performed between the Hospital for Rehabilitation of Craniofacial Anomalies (HRAC), University of São Paulo (USP), and the Educational Center of Hearing and Language Ludovico Pavoni (CEAL-LP). Ordinance Act number 402 (February 24, 2010) of the Health Ministry established the nationwide program Tele-Health Brazil, structured in a telemedicine format and aimed to quantify and expand solvability and strengthen strategies to improve family health. The Brazilian Federal Council of Audiologists published Resolution 366 (April 25, 2009) defines Telehealth as a legal audiologist practice using information technology (IT), i.e., activities and solutions based on computing resources and telecommunications, among others, in order to "assist, promote education and conduct research in Health Care."

Wesendahl (67) used technological resources designed from 2003 to describe fittings and fine-tuning of acoustic hearing aids in real environments compared with 
ideal environments, such as audiometric sound booths and audiology clinics, highlighting the claim that "the Telemedicine concept allows a highly experienced audiologist being present in remote areas without restriction of time and geographical location."

The objective of this report is to evaluate the benefits and limitations of telemedicine applied to digital hearing aid fittings and to analyze its effective application to hearing rehabilitation under current public policies in Brazil using case presentations from a test telemedicine event.

\section{METHOD}

In this work, Tele-audiology is defined as the practice of remotely fitting hearing aids. The design of the hearing aid used in this study was conducted within the Laboratory of Medical Research (LIM-32) at the Medical School, Universidade de São Paulo. The development of the Tele-audiology program was conducted by the Otorhinolaryngology Department in conjunction with the Fundação Otorrinolaringologia (Otorhinolaryngology Foundation) with funds from these units.

The participants in this study were sufficiently advised about the purpose of the study and voluntarily signed the Statement of Free Consent and Explanation (TCLE). This research was approved by the Ethics Committee of the Faculdade de Medicina (Faculty of Medicine) of the Universidade de São Paulo (number 204/ 11). This research was conducted on April 8, 2010, with an audiologist in the Otorhinolaryngology Department University de São Paulo in a specialized unit (SU) supervised by an otorhinolaryngologist and another team of 3 audiologists under the supervision of an otorhinolaryngologist at the Instituto Sul Mineiro de Otorrinolaringologia (ISMO), a Brazilian Otorhinolaryngology
Institute located in Pouso Alegre city. This unit is referred to as the remote unit (RU). Figure 1 shows a conceptual diagram of the related study here described.

Both units belong to the National Health System (SUS) certified specialized centers, in which by 2011, there were approximately 140 units (68). Pertinent data of participants are reported in Table 1.

This study included adult patients of both genders with sensorineural hearing loss (SNHL) losses from moderate to severe-moderate in at least 1 ear and moderate SNHL in both ears according to the classification proposed by Northern and Downs and cited in Bento et al. (54), with auditory deprivation for at least 3 years and who received their hearing aids through the SUS (Ordinance Act no 587 - October 7, 2004).

The Windows ${ }^{\circledR}$ operating system was used because there is a wide range of commercial applications available in the Brazilian marketplace. For the transmission and reception of audio and video over the Internet, Skype ${ }^{\mathrm{TM}}$ was chosen because it is a free application and it is used universally without reported difficulty of installation or operation. The Anyplace Control, an application for remote access, was chosen after heavy trials using a 30-day trial version, and it was found to be a simple application with

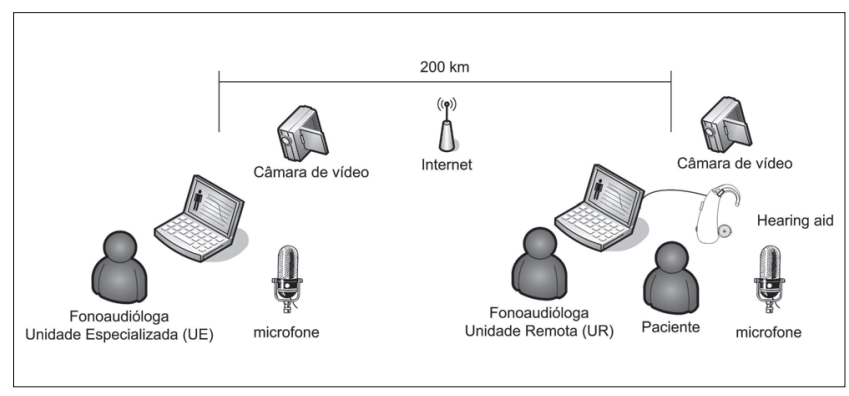

Figure 1. Conceptual diagram of the study.

Table I. Test Participants.

\begin{tabular}{|c|c|c|c|c|c|c|}
\hline Patient & Degree of loss & Gender & Age & Education & Hearing aid use (in years) & Type of hearing aid received by SUS \\
\hline D.F.B. & Moderate & Female & 61 & Elementary & । & $\begin{array}{l}\text { In-the-canal, } 4 \text { canals, maximum } \\
\text { gain of } 55 \mathrm{~dB} \text {, maker } \mathrm{A} \text {. }\end{array}$ \\
\hline E.A. & Severe-moderate & Male & 64 & Elementary & 2 & $\begin{array}{l}\text { Behind-the-ear, } 6 \text { canals, maximum } \\
\text { gain of } 58 \mathrm{~dB} \text {, Open Fit, maker B. }\end{array}$ \\
\hline J.M.S.S. & Severe-moderate & Male & 81 & Secondary & 4 & $\begin{array}{l}\text { Behind-the-ear, } 4 \text { canals, } \\
\text { maximum gain of } 6 I \mathrm{~dB} \text {, maker } \mathrm{C} \text {. }\end{array}$ \\
\hline
\end{tabular}

Notes:

1) All the hearing aids are omnidirectional only;

2) All the patients have bilateral moderate sensorineural hearing loss (SNHL);

3) Makers A, B, and C indicate the manufacturers who asked for discretion and chose not to expose their brands or models. 
a low cost. The Avast ${ }^{\circledR}$ antivirus application was used because it is free and has good market reputation. All applications used are listed in Table 2.

The gear used in this study was acquired locally, and is listed in Table 3.

For this event, only 1 behind-the-ear model Florianópolis unit was used, and its specifications are listed in Table 4. After testing, the unit was taken back to the SU.

A Remote Training Protocol (RTP) was established to provide systematic training in the use of the hearing aid and AdaptEASY. Additional informationabout the documents used in this study is listed in Table 5.

Prior to performing tests with study participants, the Internet connection reliability between the 2 units was tested and confirmed. Connection reliability performance comprises Skype ${ }^{\circledR}$, Anyplace Control, and AdaptEASY and lasts for approximately 40 minutes. In our performance tests, some delay in receiving video transmission from the SU was detected, which did not cause confusion, but did interfere with the original research goal of maximum approximation of face-to-face consultation. Because the speed of the Internet at the RU was limited to 300 kilobits per second (Kbps), which is low for maintaining audio, video, and adaptation programs simultaneously, the specialists decided to temporarily shut down the antivirus and firewall protection at the RU. As a result, the applications used during the event became faster. To avoid exposing network data to viruses, no other terminal at the RU was connected to the Internet.

All tests were thus performed on patients by the audiologists. Initially, the units were introduced trough Skype ${ }^{\circledR}$ to further review the purposes of the research. For AdaptEASY basic training, a Remote Training Protocol, Annex 1, in Portuguese, was read and understood by the RU. In the Remote Training Protocol, advanced features were omitted (e.g., the sound generator for tinnitus therapy and the Datalogging automatic recording mode of hearing aid patient behavior). That limitation in the protocol was justified in order to focus on the Tele-audiology processes instead of on the features of the hearing aid itself. Training in the research protocol lasted for approximately 30 minutes. Figure 2 shows the audiologist from SU, and in Figure 3, the audiologist appears on the notebook screen from the RU.

All patients are binaural hearing aids users but were fitted only in 1 ear in this test. This was decided because there was not a secondary objective assessment of satisfaction with the hearing aid. The hearing aid adjusted was maintained in the ear that was not aided. The adaptation sessions were carried out individually.

Table 2. List of applications used in association with this Tele-audiology event

\begin{tabular}{llcc}
\hline Name & Description & Version & Location \\
\hline adaptEASY & Fittingapplicationfor digital hearingaids & 4.00 .14 & RU \\
Skype & Internetaudioand videotransmission & SU/RU & S.2.0.155 \\
Anyplace Control & Remote ComputerAccess & 4.13 .0 .0 & SU/RU \\
Windows $^{\circledR}$ XPProfessional & OperatingSystem & SU/RU \\
Avast $^{\circledR}$ & Antivirus & EditionServicePack3 & SU/RU \\
\hline
\end{tabular}

Note. Firewall is an operating system resource.

Table 3. List of gear used in the Tele-audiology event

\begin{tabular}{lllcc}
\hline Name & Model & Maker & Location & Owner \\
\hline Notebook & Vostro I400 & Dell & $\mathrm{SU}$ & $\mathrm{SU}$ \\
Notebook & $306 \mathrm{~S}$ & PCChips & $\mathrm{RU}$ & $\mathrm{SU}$ \\
Hearingaidinterface & Hi-Pro & GNOtometric & $\mathrm{RU}$ & $\mathrm{RU}$ \\
Modem & 200E & DSLink & $\mathrm{RU}$ & $\mathrm{RU}$ \\
Modem & DPC2 I00 & WebSTAR & $\mathrm{SU}$ & $\mathrm{SU}$ \\
Router & $78.0454 \mathrm{ARB}$ & GTS & $\mathrm{SU}$ & $\mathrm{SU}$ \\
Headset & HT-30IMV & Wasta & $\mathrm{SU} / \mathrm{RU}$ & $\mathrm{SU}$ \\
Webcam & I270 & NR & $\mathrm{SU} / R U$ & $\mathrm{SU}$ \\
Webcam & I003 I & Clone & $\mathrm{SU}$ & $\mathrm{SU}$ \\
Powerspeakers & SRS68PC & Delta & $\mathrm{RU}$ & $\mathrm{RU}$ \\
\hline
\end{tabular}


Table 4. Behind-the-ear Florianópolis hearing aid specifications.

\begin{tabular}{ll}
\hline Parameter & Description \\
\hline Maximumoutput & $134 \mathrm{~dB}$ \\
Maximumgain & $65 \mathrm{~dB}$ \\
Currentdrain & $0.88 \mathrm{~mA}$ \\
Input noise & $25 \mathrm{~dB}$ \\
Batterysize & 675 \\
Batterylifespan & 480 hours \\
Comfort programs & 3 (plustelephonic coil) \\
Signal processingstrategy & WDRC \\
Numbers of channels & 8 \\
Numberofcrossovers & 7 \\
Outputlimitingstrategy & AGCo \\
Graphicequalizer & 16 frequencies \\
Beepto inform programin case & Present \\
Beeptoalertbatterylow power & Present \\
Acousticfeedbackmanager & Adaptive \\
Noise reduction & Adjustable \\
Tinnitustherapy & Tinnitus plus amplification, tinnitus only, or amplification only \\
Datalogger & Records environmentsound intensity, position of the volume control, \\
& comfortprogram, and battery voltage every I 5 minutes \\
\hline
\end{tabular}

Standard: IEC 60.118-7 (2005)

Note: 1. WDRC - wide dynamic range compression; 2. AGCo - Automatic gain control output; 3. Specifications are subject to change without notice.

Table 5. List of documents used in this study

\begin{tabular}{llcc}
\hline Name & Model & Version & Location \\
\hline TCLE & FMUSPStandard & NR & SU \\
RTP & Standard & I.I.0 & SU/RU \\
\hline
\end{tabular}

Notes: 1. TCLE - Statement of Free Consent and Explanation

2. RTP - Remote Training Protocol

3. NR - without Reference

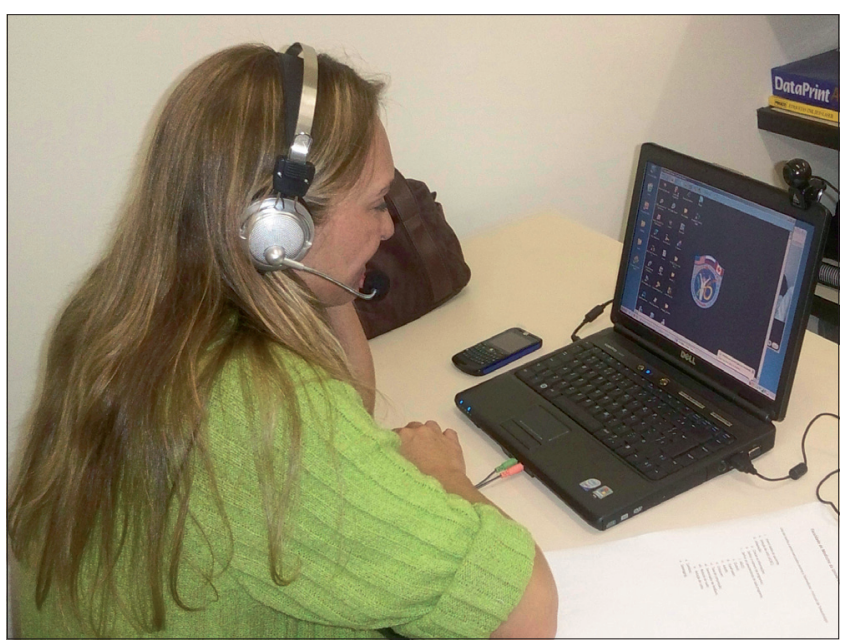

Figure 2. SU Audiologist.

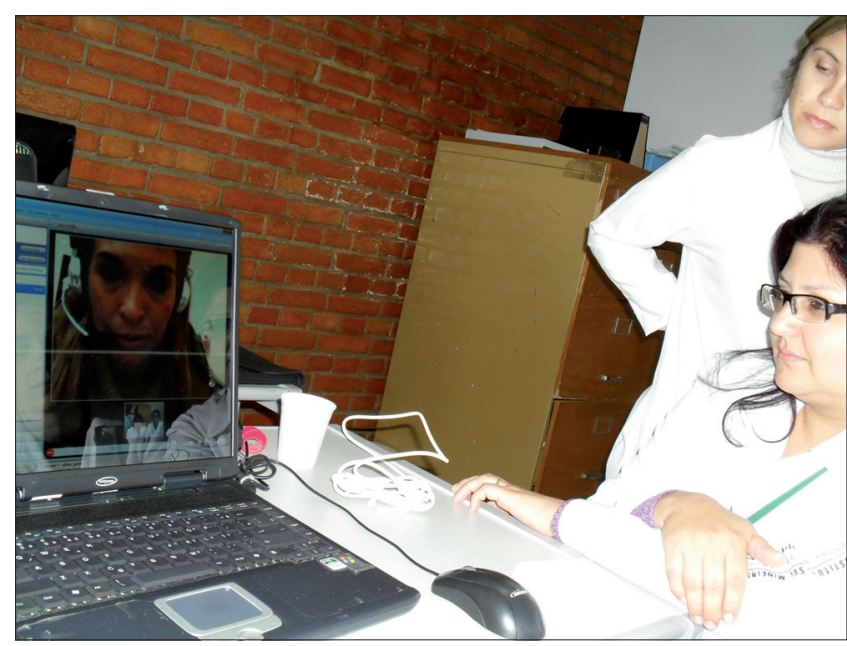

Figure 3. SU audiologist on the RU screen. 
All of the study participants were introduced to the SU audiologist while Skype connected the human element to the computerized process. The first subject (Table 3) was an in-the-canal user, and we made use of a standard dome earplug (without reference) to fit the left ear. The RU audiologists entered patient data in the AdaptEASY, and adjustments were made by the SU audiologist, who explained the criteria used to the RU audiologists. The dynamic response of the patient was transferred directly to the SU specialist through online audio, with everyone in the room using a pair of speakers. The first fitting took approximately 20 minutes.

The second participant was a behind-the-ear user with open technology (Open Fit), and again, a standard dome earplug (without reference) was used to fit the hearing aid. Similarly, the patient data were updated by RU audiologists, and adjustments were made by the SU audiologist with the hearing aid in the patient right ear. The interaction between the SU and the patient was exactly the same as that described for the first subject. This session lasted for 15 minutes.

The third patient was also a behind-the-ear user, and an ear mold of the right ear was used to fit the hearing aid. The audiologists from the RU updated patient data into the system and executed the whole process of fitting while being remotely assisted by the SU. No change in settings was proposed by the SU. This session lasted for approximately 20 minutes.

At the end of each session, the patients returned to using their regular hearing aids without any changes to the original fitting. Each patient was exempted once his/her respective session was finished. After all the procedures, the RU network was scanned for virus, and no viruses were detected. The SU was also submitted to antivirus tests, and no viruses were detected.

\section{RESULTS}

Through the Internet, RU audiologists were trained by the SU to understand the features of a hearing aid (Florianópolis) and its fitting (adaptEASY). The SU audiologist introduced and discussed the electro-acoustic hearing aid features with RU audiologists. RU audiologists highlighted the fitting program simplicity by fitting a hearing aid in a test patient without intervention of the SU. Patients interacted with both RU and SU audiologists, and they approved the remote fitting, picked out the subjective good quality of Florianópolis sound, and confirmed that remote fitting can have benefits in the fitting process.

\section{DISCUSSION}

Hearing aid manufacturers (or importers) provide hearing aids designed in developed countries that are adjusted by increasingly complex fitting programs that demand increasingly advanced computer resources and continuous training for audiologists. In this context, information technology and Tele-audiology specialists intend to provide training and forum discussions from specialized centers in any area in Brazil to ensure training uniformity and excellent outcomes. Features that can be added to the adaptEASY program include streaming training videos (forbeginners and advanced audiologists), electronic manuals, and conference calls. These resources should promote training and professional skills for the staff involved (e.g. doctors, audiologists, nurses, facilitators, and community workers).

The development of local products and services can supply solutions that better reflect the government's expectations (e.g., lower costs, jobs, and trade balance) and society's expectations (e.g., best use of public money, exchange experience, and low mobility to obtain adjustment of a hearing aid). In cases of imported hearing aids supplied by the SUS, once the warranty expires, patients may be unable to repair their hearing aids, because they are identified as retail customers and therefore subject to the same price practices as retail customers, although they are part of a wholesale practice. This is one reason why the Florianópolis was designed. Another facet of imported hearing aids lies in the complex fitting applications, meaning they are unable to provide uniform fittings because they demand specific knowledge defined by international manufacturers. Thus, more friendly and less complex fitting programs may represent a greater uniformity in fittings and, as a consequence, better results for SUS patients. Fitting programs of less complexity demand smaller investments in hardware upgrades, which may be interesting to the government as resources invested for this purpose would be less expensive and less time consuming.

One aspect that may also help in successful implementation of Tele-audiology in Brazil is the fact Brazil has no major deficiencies with regard to institutional infrastructure in the fields of otorhinolaryngology and audiology, relying on several excellence centers in these medical areas. To avoid conflicts in policy implementation, preservation of autonomy and task-sharing among activities of otorhinolaryngologists and audiologists is necessary.

The small number of SUS certified centers for hearing health policies in Brazil (approximately 140) require patients, and often their companions, to travel from their homes to these centers in detriment of their 
secular duties (paid or unpaid) and to assume responsibility for transportation and food expenses, when sometimes, it is necessary only to make adjustments in the (re) programming of their hearing aids. Such situations can result in lower incentive for the rehabilitation process even when the hearing aids are given to the population for free.

The government may extend the system once proposed in order to control the whole fitting process, including patient data (e.g. age, hearing loss degree, and etiology), demographics data (e.g., number of patients and number of patients by physicians/audiologists), and costs (e.g., hearing aid servicing and repair time, the most common problems). This information can help in continuous improvement of hearing health programs in Brazil. The hearing health program based on Tele-audiology can be implemented together with other nationwide operational units such as the Basic Health Units (UBS).

Some aspects of technological implementation deserve to be highlighted. Anyplace Control enables message exchange in chat format between the SU and the $\mathrm{RU}$, which is relevant if there are difficulties regarding transmission rate restrictions between centers. This feature is important when video cannot be maintained or when there are successive interruptions with loss during the fitting process. The delays noted in this event are inherent to the process of remote transmission and reception for 2 reasons:

1) The Windows ${ }^{\circledR}$ operating system does not operate in real time and manages tasks based on interruptions and functional priority;

2) Transmission over the Internet always presents a certain delay based on the distance covered by transmission/ reception, regardless of the speed of the Internet provider.

To solve constraint 1 , all applications should be used in structured, real-time operating systems such as $\mathrm{QNX}^{\text {ब }}$ (QNX Software Systems Corp., Ottawa, Canada), RTLinux (open source, free software), VxWorks (Wind River Systems Inc., Alameda, California, USA), and Windows ${ }^{\circledR} \mathrm{CE}$ (Microsoft Corp., Redmond, Washington, USA) among others. One way of minimizing the effects of the second restriction is to use faster service providers, ideally offering speeds more than 2 megabytes (MB) per second.

The proprietary platform chosen (Windows ${ }^{\circledR}$ ) may be replaced by an open platform (e.g., Linux) in order to offer more economical solutions that can be customized according to the specifications of individual countries based on their regulatory directions or operational requirements. The hearing aid interface (in this case the Hi-Pro) may be replaced by an application to be downloaded from the Internet and installed on the computer of the certified center in order to cut costs of equipment acquisition.

In Krumm et al. (60), 2 forms of telemedicine practice are classified: (1) synchronous, wherein communication occurs in real time (e.g., interactive video, telephone, and instant messaging), and (2) asynchronous, wherein communication occurs based on data storage and subsequent use (e.g., e-mail, fax, answering machine, pagers, web pages, web forums, and animations). The pioneering work in Brazil highlighted in this report serves both modes.

To attend the SUS at the ambulatory level, there are 4,287 UBSs spread through São Paulo state, of which 537 are in the São Paulo city extended area. There are a large number of specialized centers to attend SUS patients for fittings that are purposed as walk-in or scheduled appointment centers. These UBSs have computer hardware that may be available for the practice of Tele-audiology and must run on the same Windows ${ }^{\circledR}$ platform o ensure interoperability between all hardware system which should be one that avoids difficulties by requiring a relatively low volume of data exchange (e.g., audio and video and data session adjustment). Remote locations that permanently or temporarily do not have Internet service can be assisted through telephone services. Truly remote locations (e.g., native communities and communities in border areas) can be provided with institutional video or even with printed documents.

The change from face-to-face care to remote care demands training for those involved, and audiologists and otorhinolaryngologists should not be resistant to Teleaudiology. To ensure this, group coordinators can assemble an implementation plan with greater restrictions for places where verbal communication cannot be ruled out.

Important issues that must be considered with the practice of telemedicine include skills, competencies, and responsibilities of professionals involved, along with safety and privacy, effectiveness, efficiency, ethical aspects, restricted access, handling of patient information and whole-system information, and costs (69). The 2 additional features that should compose telemedicine are related to network stability and simultaneous functions when combined with other operating systems.

Future Tele-audiology tests should be compared with clinical results that are obtained from face-to-face sessions, contextualizing and analyzing their differences. The key aspects described in the present report demand further evaluation from a greater number of research centers due to subject plurality and various skills that will provide a better understanding of the topic. 


\section{Conclusion}

Focused on existing laws and aiming at improvement of public policies regarding hearing rehabilitation and health in Brazil, this pioneering work featured a remote hearing aid fitting with cooperation between specialists in 2 cities that are $200 \mathrm{~km}$ apart. There can be great benefits in Tele-audiology implementation in Brazil, even though the Internet is not yet available in many places in the country.

\section{REFERENCES}

1. Maheu MM, Whitten P, Allen A. E-Health, Telehealth, and Telemedicine: A guide to start-upand success. San Francisco: Jossey-Bass Inc; 2001. p.2-10.

2. DeBakey ME. Telemedicine has now come of age. Telemed J, 1995; 1(1):3-4.

3. Puentes J, Soliamn B, Roux C. Exploring telemedicine applications in gastroenterology. Proceedings of the first BMES/ EMBS Conference. Serving Humanity, Advance Technology. Page 1218. Atlanta, Georgia, Oct 13-16, 1999.

4. Chorbev I, Mihajlov M. Wireless telemedicine services as part of an integrated system for e-medicine; Electrotechnical Conference, 2008. MELECON 2008. The $14^{\text {th }}$ IEEE Mediterranean, 264-9. 5-7 May, 2008.

5. U.S. Department of Health and Human Services Telemedicine for the Medicare Population: Evidence Report/Technology Assessment Number 24. July 2001.

6. Tohme, WG, Hayes WS, Mun SK, Komo D, Meissner MC. "Designing a telemedicine platform for three different medical applications,". Proceedings of the Fourth International Conference on Image Management and Communications, 1995, vol., no., pp.86-90, 20-24 Aug 1995.

7. Belardinelli A, Franchi D, Bedini R, Ripoli A, Palagi G. Ward informative system: hospital application of telemedicine. Computers in Cardiology, 1999; 26:413-6.

8. Wootton R. The possible use of telemedicine in developing countries. Journal of Telemedicine and Telecare, 1997; 3:23-26.

9. Rahms H, Sanz M, Arredondo MT, del Pozo F. "Usability analysis of a telemedicine system for interventional cardiology," Computers in Cardiology 1995 , vol., no., pp.661-4, 10-13 Sep 1995.
10. Horsch A, Balbach T. "Telemedical information systems," Information Technology in Biomedicine, IEEE Transactions on, vol.3, no.3, pp.166-75, Sept. 1999.

11. Clarke M, Jones RW. "AIDMAN - a versatile telemedicine platform," Engineering in Medicine and Biology Society, 2001. Proceedings of the $23^{\text {rd }}$ Annual International Conference of the IEEE, vol.4, no., pp. 3496-9vol.4, 2001.

12. Glombitza G, Evers H, Hassfeld S, Engelmann U, Meinzer H-P. "Virtual surgery in a (tele-) radiology framework," Information Technology in Biomedicine, IEEE Transactions on, vol.3, no.3, pp.186-96, Sept. 1999.

13. Pradeep PV, Mishra A, Kapoor L, Basner R, Agarwal G, Agarwal A, Verma AK, Mishra SK. "Surgical sub-specialty growth in developing country: impact of telemedicine technology; a case study with endocrine surgery," e-Health Networking, Applications and Services, 2006. HEALTHCOM 2006. $8^{\text {th }}$ International Conference on, vol., no., pp. 34-9, 17-19 Aug. 2006.

14. Vasudevan S, Cleetus KJ. "Low cost telemedicine for home health care," Enabling Technologies: Infrastructure for Collaborative Enterprises, 2001. WET ICE 2001. Proceedings. Tenth IEEE International Workshops on, vol., no., pp.39-40, 2001.

15. Clarke M, Jones RW. "Technology assisted health care - an information based approach," Information Technology Applications in Biomedicine, 2003. 4th International IEEE EMBS Special Topic Conference on , vol., no., pp. 90- 3, 2426 April 2003.

16. Alqirim N. "Telemedicine for development in healthcare organizations: A myth or a fantasy," Communications, Computers and Applications, 2008. MIC-CCA 2008. Mosharaka International Conference on, vol., no., pp.xivxiv, 8-10 Aug. 2008.

17. Ramos V. "Contributions to the history of Telemedicine of the TICs," Telecommunications Conference (HISTELCON), 2010 Second IEEE Region 8 Conference on the History of , vol., no., pp.1-5, 3-5 Nov. 2010.

18. Kyriacou E, Fakas G, Pavlaki V. "Acompletely Decentralized workflow Management System for the Support of Emergency Telemedicine and Patient Monitoring," Engineering in Medicine and Biology Society, 2006. EMBS '06. 28 ${ }^{\text {th }}$ Annual International Conference of the IEEE, vol., no., pp.5663-6, Aug. 30 2006-Sept. 32006.

19. Vergados DJ, Vergados DD, Maglogiannis I. "NGL03-6: Applying Wireless DiffServ for QoS Provisioning in Mobile 
Emergency Telemedicine," Global Telecommunications Conference, 2006. GLOBECOM '06. IEEE, vol., no., pp.15, Nov. 27 2006-Dec. 12006.

20. Yoo SK, Kim BS. "Wavelet based ECG compression algorithm for emergency mobile telemedicine," SICE-ICASE, 2006. International Joint Conference, vol., no., pp.1137-8, 18-21 Oct. 2006.

21. Scheideman-Miller C, Clark PG, Smeltzer SS, Carpenter J, Hodge B, Prouty D. "Two year results of a pilot study delivering speech therapy to students in a rural Oklahoma school via telemedicine," System Sciences, 2002. HICSS. Proceedings of the 35th Annual Hawaii International Conference on, vol., no., pp. 9 pp., 7-10 Jan. 2002

22. Nakamura N. "Development of mobile audiometric test system using mobile phones," Biomedical Engineering, 2003. IEEE EMBS Asian-Pacific Conference on , vol., no., pp. 356-7, 20-22 Oct. 2003.

23. Brennan DM, Barker LM. "An interactive telemedicine system for remote speech-language pathology treatment," Engineering in Medicine and Biology Society, 2004. IEMBS '04. 26th Annual International Conference of the IEEE, vol.2, no., pp.4773-6, 1-5 Sept. 2004.

24. Vacher M, Guirand N, Serignat J-F, Fleury A, Noury N. "Speech recognition in a smart home: Some experiments for telemonitoring," Speech Technology and HumanComputer Dialogue, 2009. SpeD '09. Proceedings of the $5^{\text {th }}$ Conference on, vol., no., pp.1-10, 18-21 June 2009.

25. Ngalamou L, Rose D. "Fertility information appliance," Computer-Based Medical Systems, 2002. (CBMS 2002). Proceedings of the $15^{\text {th }}$ IEEE Symposium on, vol., no., pp. 335- 8, 2002.

26. Montesinos L, Puentes J, Madrid A, Parra A, Solaiman B, Roux C. "Integrated telediagnostic and epidemiological system to reduce cervical cancer incidence in Mexico," Engineering in Medicine and Biology Society, 2003. Proceedings of the 25th Annual International Conference of the IEEE, vol.2, no., pp. 1244-1247 Vol.2, 17-21 Sept. 2003.

27. Verma P, Ghosh AK, Huck RC, Cheng S, Chen S, Martens M, Kaul A. "Continuous Wireless Monitoring of the Cervical Dilation of a Pregnant Woman," Medical Measurements and Applications, 2008. MeMeA 2008. IEEE International Workshop on, vol., no., pp.93-6, 9-10 May 2008.

28. Stockwell SA, Glick JI. "Computerized disease vector identification keys," Military Telemedicine On-Line Today, 1995. 'Research, Practice, and Opportunities'., Proceedings of the National Forum, vol., no., pp.131-2, 27-29 Mar 1995.
29. Duy Hai Vu; Duc Thuan Nguyen. "Design of laboratory information system for healthcare in Vietnam BK-LIS," Communications and Electronics (ICCE), 2010 Third International Conference on, vol., no., pp.110-114, 11-3 Aug. 2010

30. Waterhouse E. "New horizons in ambulatory electroencephalography," Engineering in Medicine and Biology Magazine, IEEE, vol.22, no.3, pp.74-80, May-June 2003.

31. Bramanti A, Bonanno L, Celona A, Bertuccio S, Calisto A, Lanzafame P, Bramanti P. "GIS and spatial analysis for costs and services optimization in neurological telemedicine," Engineering in Medicine and Biology Society (EMBC), 2010 Annual International Conference of the IEEE, vol., no., pp.2204-7, Aug. 31 2010-Sept. 42010.

32. Rajan B, Seidmann A, Dorsey ER, Biglan KM, Reminick J. "Analyzing the Clinical and Competitive Impact of Telemedicine-Experience with Treating Parkinson Disease Patients via Telemedicine," System Sciences (HICSS), 2011 $44^{\text {th }}$ Hawaii International Conference on, vol., no., pp.1-10, 4-7 Jan. 2011

33. Zahlmann G, Kluthe S, Obermaier M, Mertz M, Mann G. "Establishment and use of a teleconsultation network in ophthalmology," Engineering in Medicine and Biology Society, 1997. Proceedings of the $19^{\text {th }}$ Annual International Conference of the IEEE, vol.2, no., pp.924-7 vol.2, 30 Oct2 Nov 1997.

34. Shengsheng Yu, Zhuo Wei, Deng, RH, Haixia Yao, Zhigang Zhao, Lek Heng Ngoh, Yongdong Wu. "A teleophthalmology system based on secure video-conferencing and white-board," e-health Networking, Applications and Services, 2008. HealthCom 2008. $10^{\text {th }}$ International Conference on, vol., no., pp.51-2, 7-9 July 2008

35. Camara JG, Santiago MDD, Rodriguez RE. "Real-time telementoring in ophthalmology," Medical Technology Symposium, 1998. Proceedings. Pacific, vol., no., pp.16672, 1998

36. Go K, Kashiwagi K, Tanabe N, Horiuchi K, Koike N. "Iterative design of Teleoperative Slit Lamp Microscopes for Telemedicine," Pervasive Computing Technologies for Healthcare (Pervasive Health), $20104^{\text {th }}$ International Conference on-NO PERMISSIONS, vol., no., pp.1-4, 22-25 March 2010

37. Guo Q, Ohsuga M, Muroi K. "Generation of high-quality images for telemedicine and tele-pathology efforts." Engineering in Medicine and Biology Society, 1998. Proceedings of the $20^{\text {th }}$ Annual International Conference 
of the IEEE, vol.3, no., pp.1288-91 vol.3, 29 Oct-1 Nov 1998.

38. Della Mea V, Roberto V, BeltramiCA. "Visualization issues in telepathology: the role of the Internet Imaging Protocol," Information Visualisation, 2001. Proceedings. Fifth International Conference on , vol., no., pp.717-22, 2001.

39. Suzuki A, Tanaka T. "Telemedicine system of pathology image using the Internet," SICE, 2007 Annual Conference, vol., no., pp.694-7, 17-20 Sept. 2007.

40. Saenz-Lechon N, Osma-Ruiz V, Godino-Llorente JI, Blanco-Velasco M, Cruz-Roldan F, Arias-LondonoJD. "Effects of Audio Compression in Automatic Detection of Voice Pathologies," Biomedical Engineering, IEEE Transactions on, vol.55, no.12, pp.2831-5, Dec. 2008.

41. KumarDK, Howard A, Mikelaitis P. "Medical e-commerce for regional Australia-Virtual Clinic Call Centre $\left(\mathrm{VC}^{3}\right)$," Information Technology Applications in Biomedicine, 2000. Proceedings. 2000 IEEE EMBS International Conference on, vol., no., pp.95-8, 2000

42. Chang T-C, Lee J-D, Wu S-J, Shih C-H, Yang M-J. "The Development and Application of the Telemedicine System in Psychiatric Counseling," Engineering in Medicine and Biology Society, 2005. IEEE-EMBS 2005. 27 $7^{\text {th }}$ Annual International Conference of the , vol., no., pp.2196-8, 1718 Jan. 2006

43. Doi K. "Computer-aided diagnosis: potential usefulness in diagnostic radiology and telemedicine" Military Telemedicine On-Line Today, 1995. 'Research, Practice, and Opportunities'., Proceedings of the National Forum, vol., no., pp.9-13, 27-29 Mar 1995.

44. Levine BA, Cleary K, Mun SK. "Deployable teleradiology: Bosnia and beyond," Information Technology in Biomedicine, IEEE Transactions on , vol.2, no.1, pp.30-4, March 1998.

45. Puech P, ChazardE, Lemaitre L, Beuscart R. "DicomWorks Teleradiology: Secure transmission of medical images over the Internet at low cost" Engineering in Medicine and Biology Society, 2007. EMBS 2007. 29 $9^{\text {th }}$ Annual International Conference of the IEEE, vol., no., pp.6705-8, 22-26 Aug. 2007.

46. Martini MG, Istepanian RSH, Mazzotti M, Philip NY. "Robust Multilayer Control for Enhanced Wireless Telemedical Video Streaming," Mobile Computing, IEEE Transactions on , vol.9, no.1, pp.5-16, Jan. 2010.

47. Edmond CV, Sluis D. "ENT surgical simulator project,"
Military Telemedicine On-Line Today, 1995. 'Research, Practice, and Opportunities', Proceedings of the National Forum, vol., no., pp.43-6, 27-29 Mar 1995.

48. Heneghan C, Sclafani AP, Stern J, Ginsburg J. "Telemedicine applications in otoloryngology" Engineering in Medicine and Biology Magazine, IEEE, vol.18, no.4, pp.5362, 79, Jul/Aug 1999.

49. Eikelboom RH, Mbao M, Atlas MD, Mitchell I, Coates H. "Compression of video-otoscope images for tele-otology: a pilot study" Engineering in Medicine and Biology Society, 2001. Proceedings of the $23^{\text {rd }}$ Annual International Conference of the IEEE, vol.4, no., pp. 3517- 20 vol.4, 2001.

50. World Health Organization-Situation review and update on deafness, hearing loss and intervention programmes: proposed plans of actions for prevention and alleviation of hearing impairment in countries of the South-East Asia Region. SEA-Deafness-10.; Dec. 2007.

51. Smith AW. 2001. WHO activities for prevention of deafness and hearing impairment in children. Scandinavian Audiology. 30(2):93-100. http://www.informaworld.com/ 10.1080/010503901750166808; (accessed 13 April 2010).

52. World Health Organization - Deafness and hearing impairment survey: Report of the consultative meeting of principal investigators. SEA-Deaf-4; July 2001.

53. Penteado SP, Bento RF. Designing of a digital behindthe-ear hearing aid to meet the World Health Organization requirements.doi: 10.1177/1084713810380934 TRENDS AMPLIF June 2010 vol. 14 no. 2 64-72.

54. Bento RF, Miniti A, Marone SAM. Tratado de otologia. 1a ed. São Paulo: EDUSP; 1998.

55. Miniti A, Bento RF, Butugan O. Otorrinolaringologia clínica e cirúrgica. São Paulo: Atheneu; 2000.

56. WHO - World Health Organization. Guidelines for hearing aids and services for developing countries. Sep 2004.

57. Goulios H, Patuzzi RB. Audiology education and practice from an international perspective. International Journal of Audiology, 2008; 47(10):647-64.

58. Kokesh J, Ferguson AS, Patricoski C, LeMaster B. Traveling an audiologist to provide otolaryngology care using store-and-forward telemedicine. Telemedicine Journal \& E-Health, 2009; 15(8):758-63.

59. Krumm M, Ribera J, Schmiedge J. Using a telehealth medium for objective hearing testing: implications for 
supporting rural universal newborn hearing screening programs. Semin Hear, 2005; 26(1):3-12.

60. Krumm M. Audiology telemedicine. JTelemed Telecare, 2007; 13(5):224-9.

61. Bloom S. New technologies can link hearing care providers with distant patients. The Hearing Journal, 1999; 52(7):21-30.

62. Givens GD, Blanarovich A, Murphy T, Simmons S, Blach D, Elangovan S. Internet-based tele-audiometry system for the assessment of hearing: a pilot study. Telemed J E Health, 2003; 9(4):375-8.

63. Givens GD, Elangovan S. Internet application to teleaudiology_-nothin' but net". Am J Audiol, 2003; 12(2):5965.

64. Ramos A, Rodríguez C, Martinez-Beneyto P, Perez D, Gault A, Falcon J, et al. Use of telemedicine in the remote programming of cochlear implants. Acta Oto-Laryngologica, 2009; 129(5):533-40.
65. XIX Congresso Internacional da International Federation of Oto-rhino-laringological Societes (IFOS), São Paulo, 31 de maio - 4 junho de 2009. Remote fitting of cochlear implant system.

66. Zumpano CE, Bevilacqua MC, Frederigue-Lopes NB, Costa AO. Programação remota dos sistemas de implante coclear. Rev. Soc. Bras. Fonoaudiol, 2009; 14(4):539-46.

67. Wedendahl T. Hearing aid fitting: application of Telemedicine in Audiology. International Tinnitus Journal, 2003; 9(1):56-8.

68. http://portal.saude.gov.br/portal/arquivos/pdf/ servicos_auditiva.pdf, visited on october 12, 2011.

69. Bauer JC; Ringell MA. Telemedicine and the reinvention of Healthcare. New York: McGrawn- Hill; 1999. p.157-81. 